\title{
Antimycobacterial susceptibility evaluation of rifampicin and isoniazid benz-hydrazone in biodegradable polymeric nanoparticles against Mycobacterium tuberculosis H37Rv strain
}

This article was published in the following Dove Press journal:

International Journal of Nanomedicine

\author{
Sushruta S Hakkimane \\ Vishnu Prasad Shenoy ${ }^{2}$ \\ Santosh L Gaonkar ${ }^{3}$ \\ Indira Bairy ${ }^{4}$ \\ Bharath Raja Guru',5 \\ 'Department of Biotechnology, \\ Manipal Institute of Technology, \\ Manipal Academy of Higher \\ Education, Manipal, India; ${ }^{2}$ Department \\ of Microbiology, Kasturba Medical \\ College, Manipal Academy of Higher \\ Education, Manipal, India; ${ }^{3}$ Department \\ of Chemistry, Manipal Institute of \\ Technology, Manipal Academy of \\ Higher Education, Manipal, India; \\ ${ }^{4}$ Department of Microbiology, Melaka \\ Manipal Medical College, Manipal \\ Academy of Higher Education, \\ Manipal, India; ${ }^{5}$ Manipal McGill Center \\ for Infectious Diseases, Manipal \\ Academy of Higher Education, \\ Manipal, India
}

Introduction: Tuberculosis (TB) is the single largest infectious disease which requires a prolonged treatment regime with multiple drugs. The present treatment for TB includes frequent administration of a combination of four drugs for a duration of 6 months. This leads to patient's noncompliance, in addition to developing drug-resistant strains which makes treatment more difficult. The formulation of drugs with biodegradable polymeric nanoparticles (NPs) promises to overcome this problem.

Materials and methods: In this study, we focus on two important drugs used for TB treatment - rifampicin (RIF) and isoniazid (INH) - and report a detailed study of RIF-loaded poly lactic-co-glycolic acid (PLGA) NPs and INH modified as INH benz-hydrazone (IH2) which gives the same therapeutic effect as INH but is more stable and enhances the drug loading in PLGA NPs by 15 -fold compared to INH. The optimized formulation was characterized using particle size analyzer, scanning electron microscopy and transmission electron microscopy. The drug release from NPs and stability of drug were tested in different $\mathrm{pH}$ conditions.

Results: It was found that RIF and IH2 loaded in NPs release in a slow and sustained manner over a period of 1 month and they are more stable in NPs formulation compared to the free form. RIF- and IH2-loaded NPs were tested for antimicrobial susceptibility against Mycobacterium tuberculosis H37Rv strain. RIF loaded in PLGA NPs consistently inhibited the growth at 70\% of the minimum inhibitory concentration (MIC) of pure RIF (MIC level $1 \mu \mathrm{g} / \mathrm{mL}$ ), and pure IH2 and IH2-loaded NPs showed inhibition at MIC equivalent to the MIC of INH $(0.1 \mu \mathrm{g} / \mathrm{mL})$.

Conclusion: These results show that NP formulations will improve the efficacy of drug delivery for TB treatment.

Keywords: rifampicin nanoparticles, isoniazid hydrazone, drug susceptibility, Mycobacterium tuberculosis

\section{Introduction}

Tuberculosis (TB) is a highly communicable disease which is the principal cause of mortality worldwide. In spite of immense success achieved by the antitubercular drugs (ATDs), it still continues to inflict major challenges on global health care. ${ }^{1}$ The World Health Organization declared TB as a global public health emergency in $1993,{ }^{2}$ and a goal was set to eliminate TB by $2050 .^{3}$ As per statistics, over eight million people suffer from active TB, and approximately two million deaths occur every year. The treatment for TB includes administration of multidrug combination of rifampicin (RIF), isoniazid (INH), pyrazinamide and ethambutol for 2 months
Correspondence: Bharath Raja Guru Department of Biotechnology, Manipal Institute of Technology (MIT), Manipal Academy of Higher Education, Manipal 576/04, India

Tel +91 8202924321

Email bharath.guru@manipal.edu 
followed by the administration of RIF and INH for 4 months. The drug-sensitive TB is effectively treated for 6 months by multidrug therapy. Since this therapy is carried out for a long duration, chances of patients discontinuing the course before the infection is completely cleared are high, and this compromises the patient's compliance and adherence to treatment. The failure of treatment could lead to the appearance of multidrug-resistant and extensively drug-resistant TB. The drug-resistant strains pose challenges for treatment and eradication of TB. ${ }^{5}$

The presence of active or dormant bacteria within the host lung lesions makes the treatment difficult due to its complex existence and survival. This disrupts the host's immune system and prevents the drugs from reaching the sites of infection. ${ }^{4,6}$ Due to the urgent need and severity of the disease in the beginning and middle of the 20th century, most of the drugs used for TB have been in use before pharmacokinetic and pharmacodynamic studies of drugs were done, and almost all the drugs were introduced 60 years ago. ${ }^{7,8}$ During the drug development process, correlations between the therapeutic concentration and the drug concentration in plasma after administration were not given due consideration. This gave rise to a suboptimized dose - a problem that is now extensively addressed for RIF. ${ }^{7,8}$

RIF and INH, the two most potent ATDs, are administered for at least 6 months - RIF hinders the gene transcription by inhibiting DNA-dependent RNA polymerase..$^{9-11}$ The INH is a prodrug, and the mycobacterial enzyme catalase-peroxidase converts it into its active form, isonicotinic acid, which acts on the cell walls of Mycobacterium tuberculosis (Mtb) and inhibits the synthesis of mycolic acids. ${ }^{12-16}$

Though RIF and INH are very effective as TB drugs, there are many challenges to treat patients with an optimized dosage. The poor solubility and low bioavailability of RIF obstructs accomplishment of therapeutic concentrations at the targeted site. Though concentration of RIF is directly proportional to its bactericidal activity, long and continuous therapy induces hepatotoxicity. ${ }^{17-20}$ The INH induces peripheral neuropathies, and its overdosage results in seizures. ${ }^{21}$ The current ATDs are effective, provided proper drug delivery strategy is employed for TB treatment. There is scope for modification of old drugs and development of new delivery systems ${ }^{22,23}$ which can improve efficiency and reduce toxicity.

The delivery of ATDs with nanoparticle (NP)-based controlled-delivery devices is one of the promising approaches. ${ }^{24}$ Several reports have been published on the advantages of NP drug delivery systems for infectious diseases. ${ }^{25-27}$ Some of the drug delivery systems have been accepted for clinical treatment of different infectious diseases, while few others are currently under different phases of clinical and preclinical trials. ${ }^{28,29}$ The polymeric NPs offer unique benefits to achieve a slow and sustained release that has the potential to treat chronic diseases like TB. ${ }^{30}$ Our study is focused on strengthening the current treatment regime for $\mathrm{TB}$ which is not just complex but also requires a prolonged treatment with multiple drugs. In this study, we prepared a nanoformulation of the two most effective first-line drugs, RIF and INH, which are used in both the phases of the 6-month TB therapy. Since INH is small and a highly hydrophilic molecule, it has a low cellular penetration ${ }^{31}$ and also low drug loading efficiency in nanoformulation using hydrophobic US Food and Drug Administration approved polymers like poly lactic-co-glycolic acid (PLGA). This has led to a considerable hindrance in effective treatment with INH. To overcome this, INH was modified into INH benz-hydrazone (IH2) by adding a hydrophobic moiety called benzaldehyde, a commonly used food additive, using Schiff base reaction. The newly formed IH2 is encapsulated in PLGA polymer, and its encapsulation in polymer is increased around 15-fold compared to INH encapsulation in PLGA. The NPs of RIF and IH2 were characterized using dynamic light scattering (DLS), scanning electron microscopy (SEM) and transmission electron microscopy (TEM). In vitro drug release kinetics from PLGA NPs and stability of RIF and IH2 were analyzed at $37^{\circ} \mathrm{C}$ and in different $\mathrm{pH}$ buffers in order to simulate the diseased site of the human body. The infected bacilli may be found in extracellular environment or intracellular part of macrophages. To mimic both the conditions, in vitro drug release was conducted at endosomal $\mathrm{pH}$ (approximately 5.2), lysosomal $\mathrm{pH}$ (around 4.5-5), blood pH (7.4) and stomach pH (around 2-3).

To check the effectiveness of the nanoformulation, antimicrobial susceptibility test was carried out using Becton Dickinson Detection (BACTEC ${ }^{\text {TM}}$ ) Mycobacterium Growth Indicator Tube (MGIT ${ }^{\mathrm{TM}}$ ) 960 instrument against H37Rv strain through a procedure similar to the one carried out for patients' samples in diagnostic laboratory. Currently, MGIT'T 960 is considered the benchmark in liquid culture system and is introduced for diagnosis and drug susceptibility test of Mtb under Revised National TB Control Programme in India. ${ }^{32,33}$ Here, we report NPs encapsulated with RIF inhibited Mtb $\mathrm{H} 37 \mathrm{Rv}$ strain at 30\% reduced minimum inhibitory concentration (MIC). The modified compound IH2 was found to be stable and restricted the growth of Mtb at its MIC level.

This study provides evidence that drugs encapsulated in NPs aid in inhibition of Mtb growth at a substantially lower concentration and are also more stable in different $\mathrm{pH}$ conditions compared to the pure drugs. The results 
provide the scope to take up further studies to improve the treatment of TB.

\section{Materials and methods Materials}

PLGA 50:50 (MW: 1,00,000-1,20,000 Da) was procured from Durect Corporation (Birmingham, AL, USA), RIF from Duchefa-Genetix Biotech, acetonitrile and methanol from Merck Millipore (Billerica, MA, USA) and INH, benzaldehyde, ethyl alcohol and trifluoroacetic acid (TFA) from Loba Chemie (Mumbai, India). The rest of the reagents and chemicals used were of high-performance liquid chromatography (HPLC) grade. Polyvinyl alcohol (PVA) (87\%-89\% hydrolyzed, MW: 1,20,000 Da) was procured from Thomas Baker (Mumbai, India). Middlebrook 7H9 medium, antimicrobial mixture MGIT ${ }^{\mathrm{TM}}$ PANTA ${ }^{\mathrm{TM}}$ and MGIT ${ }^{\mathrm{TM}}$ growth supplement oleic acid-albumin-dextrose-catalase (OADC) were procured from Becton Dickinson (Franklin Lakes, NJ, USA). Löwenstein-Jensen (LJ) medium, blood agar media, Petri plates and disposable loops were procured from HiMedia (Mumbai, India). The cell culture requirements, Ham's F-12 medium, Dulbecco's Modified Eagle's Medium (DMEM; HiMedia), fetal bovine serum (FBS) and Coumarin 6 were procured from Sigma-Aldrich (St. Louis, MO, USA).

\section{Preparation of RIF- and IH2-loaded PLGA NPs}

The RIF-encapsulated NPs were formulated using PLGA 50:50 by modified single-emulsion solvent evaporation method. ${ }^{34}$ In brief, $90 \mathrm{mg}$ of PLGA polymer and $45 \mathrm{mg}$ of RIF were dissolved in $2 \mathrm{~mL}$ of chloroform, and this mixture was added to $12 \mathrm{~mL}$ of $2.5 \%$ PVA solution. The mixture was sonicated at $80 \%$ amplitude for 6-8 minutes with a pulse time of 10 seconds using a tip sonicator (Q-700 Ultrasonication Device; Q Sonica, Newtown, CT, USA) under an ice bath. The emulsion obtained was left for organic solvent evaporation on a magnetic stirrer (SCHOTT Instrument, Mainz, Germany) for 12-16 hours. PVA was removed by washing three times with pure water. The supernatant was used for surface tension measurement using a tensiometer, and the particles were resuspended in $5 \mathrm{~mL}$ of distilled water. These dispersed NPs were frozen overnight at $-80^{\circ} \mathrm{C}$ and then subjected to lyophilization for 48 hours to obtain dry powder of NPs. IH2-encapsulated NPs, Coumarin NPs and blank NPs (without drug) were also prepared using the same method. The NPs obtained were stored at $-20^{\circ} \mathrm{C}$.

\section{Surface tension measurement}

The NPs-washed liquid was collected during each stage of washing, and its surface tension was measured using a tensiometer. The surface tension of $2.5 \%$ PVA and pure water was measured and then compared with the surface tension of washed liquid to confirm the removal of PVA from the NPs.

\section{Synthesis of $\mathrm{IH} 2\left(\mathrm{~N}^{\prime}\right.$ - benzylideneisonicotinohydrazide)}

An equimolar mixture of INH and benzaldehyde was refluxed for 2 hours at $70^{\circ} \mathrm{C}$ in isopropyl alcohol medium in the presence of catalytic amount of acetic acid. The reaction progression was monitored by thin-layer chromatography. Soon after the completion of the reaction, the mixture was diluted with water and stirred for an hour, and the solid formed was filtered and recrystallized from ethanol to obtain white crystalline powder to yield $85 \%$ of the desired product. The scheme of synthesis is shown in Figure 1. The product was characterized by ${ }^{1} \mathrm{H}$ nuclear magnetic resonance (NMR), ${ }^{13} \mathrm{C}$ NMR (Bruker $400 \mathrm{MHz}$ NMR spectrophotometer, Fallanden, Switzerland) and Fourier transform infrared spectroscopy (FTIR; Shimadzu-8300 FTIR spectrometer, Kyoto, Japan). The melting points of the compound were determined in open capillary tubes and were uncorrected.

\section{Measurement of particle size and zeta potential}

The particle size of the NPs was estimated using DLS method by Horiba Scientific SZ-100 (Z-type) version 2.00 (Kyoto, Japan). The lyophilized samples of NPs were redispersed with Millipore water by sonicating (Ultra Bath Sonicator; Grant Instruments, Cambridge, UK) for 30 seconds under
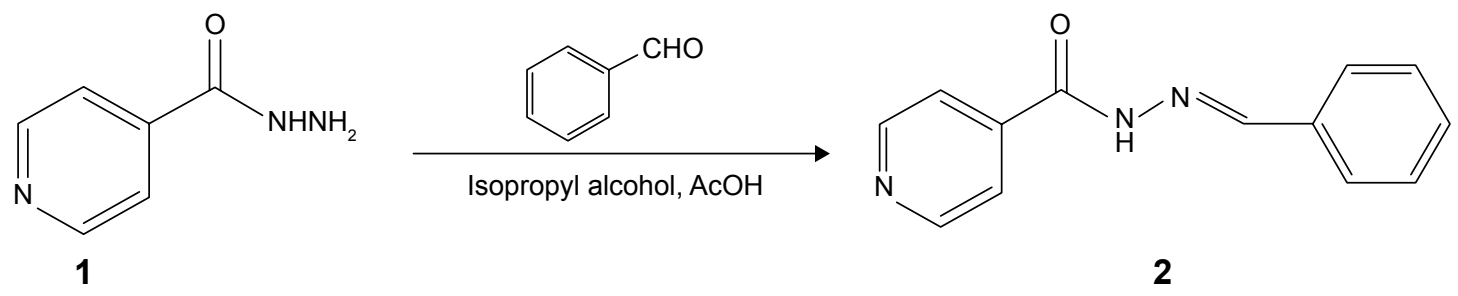

Figure I Synthetic scheme of $\mathrm{INH}$ to $\mathrm{IH} 2$.

Abbreviations: $\mathrm{IH} 2$, INH benz-hydrazone; $\mathrm{INH}$, isoniazid.

International Journal of Nanomedicine 2018:13 
an ice bath and then analyzed by particle size analyzer. The experiments were performed at a scattering angle of $90^{\circ}$ at $25^{\circ} \mathrm{C}$. The zeta potential of the NPs was measured using electrophoretic cell of the instrument. The average values were reported.

\section{Scanning electron microscopy}

The characterization of the NPs shape and morphology was done using SEM. The samples in the dry powder form were fixed on the specimen stub and coated with a thin film of gold using sputter coating instrument. SEM was performed using a scanning electron microscope (JEOL JSM-6380LA; Tokyo, Japan) at an acceleration voltage ranging from 10 to $20 \mathrm{kV}$ under a magnification of $\times 15,000-30,000$.

\section{Transmission electron microscopy}

TEM analysis was done to observe the encapsulation of drug inside the NPs and the morphology of the NPs. The particles were dispersed in water, placed on a grid and kept overnight for drying, and then the sample was observed using a transmission electron microscope (JEOL JEM-2100).

\section{RP-HPLC and LC/MS methods RP-HPLC method}

Analysis of all the samples of drugs was done using reverse-phase HPLC (RP-HPLC) system (Shimadzu Model No LC-20AD), equipped with an autosampler (SPD-20A) and a UV detector, integrated with LC Solution Software (Kyoto, Japan). Phenomenex ${ }^{\circledR}$ Luna C18 columns of length 250 and $150 \mathrm{~mm}$ were used for analysis. All the chemicals and reagents used were of HPLC grade.

A rapid and sensitive RP-HPLC method was developed using Phenomenex ${ }^{\circledR}$ Luna C18 column (250 mm length, $5 \mu \mathrm{m}, 100 \AA$ ). Acetonitrile and water with $0.1 \%$ TFA were used as solvent $\mathrm{A}$ and $\mathrm{B}$ of mobile phase, respectively. An isocratic elution of $A$ and $B(65: 35)$ at a flow rate of $1.0 \mathrm{~mL} / \mathrm{min}$ was carried out, and UV absorbance at $290 \mathrm{~nm}$ was used for detection of RIF.

The statistical validation parameters such as linearity, accuracy and precision were checked according to the International Council for Harmonisation of Technical Requirements for Pharmaceuticals for Human Use (ICH) (Q2R1) guidelines.

The stock solution of RIF was prepared by dissolving $10 \mathrm{mg}$ of the drug in $10 \mathrm{~mL}$ of acetonitrile to make the final concentration of $1 \mathrm{mg} / \mathrm{mL}$. The calibration graph was constructed with nine concentrations ranging from $1 \mu \mathrm{g} / \mathrm{mL}$ to $140 \mu \mathrm{g} / \mathrm{mL}$.

\section{System suitability}

Once the HPLC system was stabilized, three blanks followed by five replicates of a single-calibration standard solution of RIF were injected to check the system suitability.

To calculate the limit of detection (LOD) and limit of quantitation (LOQ) from the stock solution, 36 different concentrations of RIF in acetonitrile were prepared ranging from $10 \mathrm{ng} / \mathrm{mL}$ to $140 \mu \mathrm{g} / \mathrm{mL}$. The triplicates of each concentration of these solutions were injected. The LOD and LOQ were calculated as per the ICH guidelines using the following equation:

$$
\mathrm{LOD}=3 \sigma / \mathrm{S} \text { and } \mathrm{LOQ}=10 \sigma / \mathrm{S}
$$

where $\sigma=$ standard deviation of the peak area and $\mathrm{S}=$ average of the slope of the corresponding calibration curve.

The RP-HPLC method development and validation for INH have been described. ${ }^{35}$ The IH2 was estimated using RP-HPLC with Phenomenex ${ }^{\circledR}$ Luna C18 column (4.6×150 mm, $5 \mu \mathrm{m}, 100 \AA)$. The mobile phase used was 60:40 methanol and water in isocratic mode, and UV absorbance was detected at $268 \mathrm{~nm}$.

\section{Simultaneous estimation of $\mathrm{INH}$ and $\mathrm{IH} 2$}

It was required to estimate $\mathrm{INH}$ and $\mathrm{IH} 2$ simultaneously to check the stability of IH2 with respect to INH and find out whether transition occurred from IH2 to INH. The same column was used to estimate them simultaneously. The solvents water and methanol at concentrations varying from 90:10 to 10:90 were eluted in gradient flow mode for 12 minutes and then slowly brought back to initial concentration in a duration of 20 minutes.

\section{LC/MS method}

The molecular weight and purity of IH2 were analyzed using liquid chromatography-mass spectrometry (LC/MS). The mass spectra were recorded on an Agilent 6520 AccurateMass Q-TOF LC/MS system at a gas temperature of $350^{\circ} \mathrm{C}$. Phenomenex ${ }^{\circledR}$ Luna C18 column $(150 \times 4.60 \mathrm{~mm}, 5 \mu \mathrm{m}$, $200 \AA$ ) was used. Water and acetonitrile with $0.1 \%$ formic acid were used as mobile phase, keeping the sample injection volume at $2 \mu \mathrm{L}$, and the run time was set at 20 minutes.

\section{Drug encapsulation inside the NPs}

The drug encapsulation inside the NPs was measured by the amount of drug encapsulated per milligram of NPs. To calculate drug encapsulation, a specific amount of NPs was weighed and dispersed in a calculated amount of 
methanol. ${ }^{36}$ The vials were kept on a rocker shaker for 2 days to release the drug from the polymer matrix. The vials were centrifuged at 13,000 rpm for 20 minutes, and the supernatant was analyzed using RP-HPLC to calculate the exact amount of drug encapsulated in NPs. The experiment was conducted in triplicates, and the average value was considered for the calculation.

\section{Drug stability studies}

The RIF, INH and IH2 were analyzed for their stability at different $\mathrm{pHs}$ using $\mathrm{pH} 7.4,4.5$ and 2.5 buffers. The appropriate dilutions were made and analyzed by RP-HPLC. The samples containing the drugs were kept on a rotary shaker at $37^{\circ} \mathrm{C}$ and room temperature and analyzed at different time intervals. A specific amount of drug was weighed and dissolved in a known amount of buffer of $\mathrm{pH} 7.4,4.5$ and 2.5 and centrifuged at 13,000 rpm to separate the undissolved drug. The supernatant was used for the stability test. All the samples were prepared in triplicates. The initial readings were taken for all samples by using the developed RP-HPLC method. The readings were taken at particular intervals of time after incubating the samples at $37^{\circ} \mathrm{C}$ and also at room temperature to check the drug stability.

\section{In vitro drug release studies}

The PLGA-RIF- and PLGA-IH2-loaded NP formulations were assessed for in vitro drug release studies. Three milligrams of lyophilized NPs was weighed in vials and dispersed in different $\mathrm{pH}$ buffer solutions in triplicates $(\mathrm{pH} 2.5,4.5$, 5.2 and 7.4). These vials were incubated at $37^{\circ} \mathrm{C}$ on a rotary shaker to simulate the body condition. At selected time intervals, the dispersion was ultracentrifuged at 10,000 rpm for 20 minutes. The supernatant was collected from each tube and analyzed using RP-HPLC. The NPs were redispersed in a known amount of buffer and kept in a rotary shaker. This procedure was continued till the last reading of the study. The result was calculated as the cumulative amount of drug released from the NPs with respect to time in days and hours.

\section{Antimycobacterial susceptibility test (AST) for PLGA-RIF and PLGA-IH2 NPs using MGITTM 960}

All the mycobacterial works were done in a biosafety cabinet type 2 b using biosafety level 3 practices in the mycobacteriology section. The Mtb strain H37Rv was procured from the National Institute for Research in Tuberculosis (NIRT) (Chennai, India) and maintained at Department of Microbiology, Kasturba
Medical College (KMC), Manipal. It was grown in modified Middlebrook $7 \mathrm{H} 9$ broth medium.

For AST, the Mtb H37Rv was cultured in the MGITTM tubes. Fifteen milliliters of MGIT ${ }^{\mathrm{TM}}$ growth supplement was added to a lyophilized mixture of antimicrobial agents PANTA $^{\text {TM }}$ and dissolved completely by proper mixing. From this mixture, $0.8 \mathrm{~mL}$ was taken and added to MGIT ${ }^{\mathrm{TM}}$ tube in a sterile condition using an adjustable pipette. From this wellmixed stock culture, $0.5 \mathrm{~mL}$ was added to an appropriately labeled MGIT ${ }^{\mathrm{TM}}$ tube. The tubes were tightly recapped immediately, and then mixed by slowly inverting them multiple times. The tubes were wiped from outside with a disinfectant, and the inoculated tubes were incubated at $37^{\circ} \mathrm{C}$.

All inoculated MGIT ${ }^{\mathrm{TM}}$ tubes were individually scanned and loaded into the BACTEC ${ }^{\text {TM }}$ MGIT $^{\text {TM }} 960$ instrument. The cultures were observed automatically every 60 minutes by the instrument, and the positives were signaled by visual and acoustic indications on the basis of specific growth algorithms. The incubated tubes were monitored until the instrument flagged the positivity of these cultures. ZiehlNeelsen smears were performed with the positive cultures, to check the presence of acid-fast bacilli, and MPT64 antigen kit was also used to confirm the Mtb. Further, purity test was done by subculturing onto blood agar plates and incubating at $37^{\circ} \mathrm{C}$ for 48 hours to check for other bacterial contamination.

Once the positive growth was confirmed by the instrument and purity of the growth culture was tested, AST was performed on the fifth day. The inoculum of known dilutions was prepared using the positive growth culture and sterile saline. All the tubes were labeled properly, and $0.8 \mathrm{~mL}$ growth supplement of OADC was added to the MGIT ${ }^{\text {TM }}$ tubes containing $7 \mathrm{~mL}$ of broth base. A known concentration of RIF (pure form) or RIF-loaded NPs or NPs without drug was added to appropriately labeled MGIT'T tubes. The RIF concentration was made up to $1.0 \mu \mathrm{g} / \mathrm{mL}$ (MIC of RIF). The IH2 concentration was made up to $0.16 \mu \mathrm{g} / \mathrm{mL}$ (equivalent to MIC of INH $0.1 \mu \mathrm{g} / \mathrm{mL}$ ). ${ }^{37,38}$ The control (H37Rv strain inoculum without any NPs/drug) and treatment tubes were inoculated with a known, calculated amount of inoculum. After inoculation, the tubes were placed into the BACTEC ${ }^{\text {TM }}$ MGIT $^{\text {TM }} 960$ instrument and maintained at $37^{\circ} \mathrm{C}$, and the growth was monitored. After analyzing the results, sensitivity tests were further carried out by reducing the drug concentrations. The next set of experiments were performed with reduced drug concentration and drug encapsulated in NPs with the drug concentration equivalent to $0.80,0.75,0.70,0.65,0.60,0.50$ and $0.40 \mu \mathrm{g} / \mathrm{mL}$, and each of the experiments was repeated six times. Based on 
the amount of drug loading within the NPs, the final drug concentrations for all sets of NPs were serially diluted to get the required concentrations just before the test. Sterile water was used to disperse the NPs, and all the dilutions were done in biosafety cabinet to maintain sterility. The inoculated tubes were loaded into the instrument and maintained at $37^{\circ} \mathrm{C}$, and the growth was monitored. Similarly, AST of IH2 and IH2 NPs was performed.

\section{Reinoculation onto LJ media}

Once the results were obtained from MGIT ${ }^{\mathrm{TM}}$ liquid culture, the experiment proceeded with inoculation onto LJ egg-based solid medium for the confirmation of results. The LJ slants were prepared in screw cap bottles. From each sample, $0.2 \mathrm{~mL}$ was inoculated onto LJ solid media. The slants were incubated at $37^{\circ} \mathrm{C}$ for $6-12$ weeks for visible appearance of the colonies.

\section{Study of cytotoxic effect of drug concentrations on A549 and RAW 264.7 cell lines}

A549 lung epithelial cell lines and RAW 264.7 macrophage cell lines were procured from NCCS (Pune, India). They were maintained in F-12K medium and DMEM, respectively, with $10 \%$ FBS and $1 \%$ antimicrobial mixture. The cells were grown in an incubator maintained at $37^{\circ} \mathrm{C}$ and $5 \% \mathrm{CO}_{2}$. The cells were seeded in 96-well plates at a density of 5,000 cells per well and incubated overnight for adherence. The samples added were pure RIF ( $1 \mu \mathrm{g} / \mathrm{mL}, \mathrm{MIC})$, RIF-loaded NPs ( $1 \mu \mathrm{g} / \mathrm{mL}$ of RIF in NPs), pure IH2 $(0.16 \mu \mathrm{g} / \mathrm{mL})$, IH2-loaded NPs $(0.16 \mu \mathrm{g} / \mathrm{mL}$ of IH2 in NPs $)$ and plain NPs. After 24 and 48 hours of treatment, MTT assay was carried out using a known protocol. ${ }^{39}$ The supernatant was removed from each well after 24 or 48 hours, and MTT was added $(5 \mathrm{mg} / \mathrm{mL})$ to the cells and kept at $\mathrm{CO}_{2}$ incubator for 4 hours. After incubation, MTT was removed, and $100 \mu \mathrm{L}$ of DMSO was added to each well of 96-well plates. The UV absorbance was read at $570 \mathrm{~nm}$ using a multiplate reader. The cell viability of each sample was recorded.

\section{Results}

\section{Particle size distribution and zeta potential of NPs}

The mean particle size of PLGA NPs without drugs was found to be $184 \pm 6.8 \mathrm{~nm}$, polydispersity index (PDI) $0.4 \pm 0.07$ and mean zeta potential $-29 \pm 18 \mathrm{mV}$ as shown in particle size distribution graph (Figures 2 and $\underline{\mathrm{S} 1}$ ). The mean particle size of PLGA-RIF NPs was found to be $249 \pm 40 \mathrm{~nm}$, PDI 0.4 and mean zeta potential $-43 \pm 12 \mathrm{mV}$ (Figures 3 and $\underline{\mathrm{S} 2}$ and $\underline{\mathrm{S} 3}$ ).
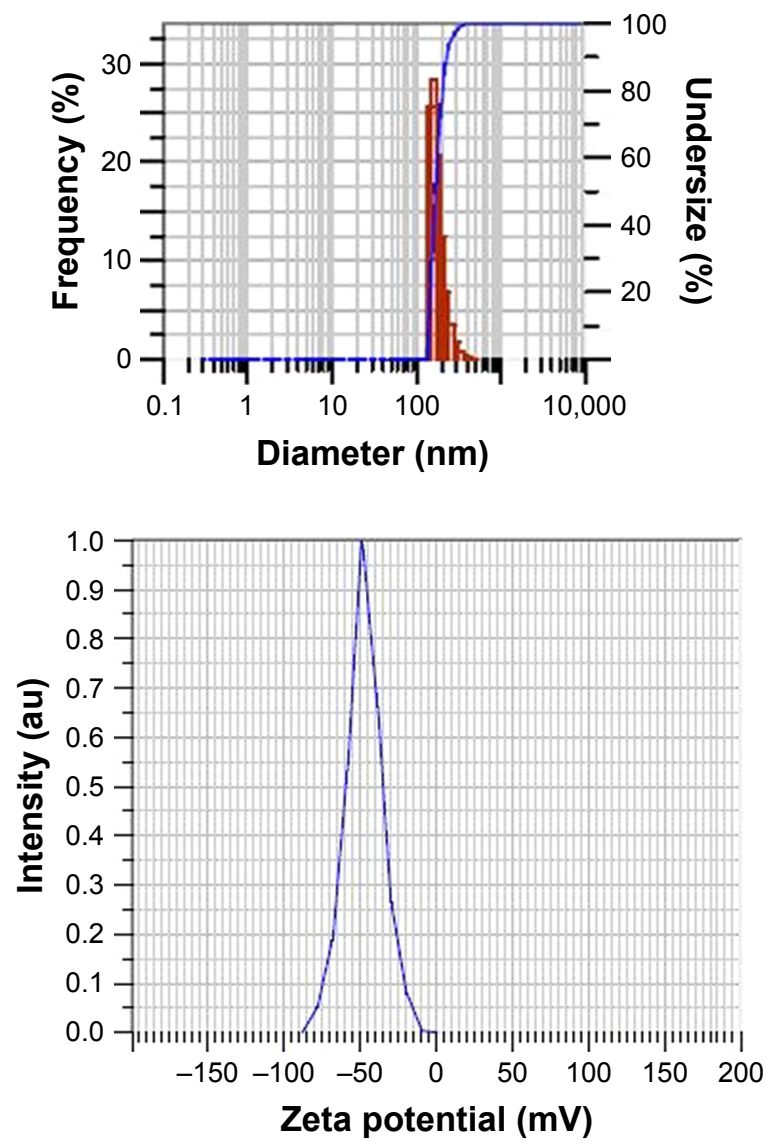

Figure 2 Particle size distribution and zeta potential graphs of plain PLGA nanoparticles (control).

Abbreviation: PLGA, poly lactic-co-glycolic acid.

The mean particle size of IH2 NPs was $309 \pm 17 \mathrm{~nm}$, PDI $0.2 \pm 0.1$ and zeta potential $-26 \pm 6 \mathrm{mV}$ which confirms good stability (Figures 4 and $\underline{\mathrm{S} 4}$ and $\underline{\mathrm{S} 5}$ ).

\section{Surface tension measurement}

The surface tension of water and 2.5\% PVA measured was $70.97 \pm 2$ and $47 \pm 2 \mathrm{mN} / \mathrm{m}$, respectively, at room temperature. The surface tension of supernatant of the third and final wash during the NP preparation was found to be $63.12 \pm 3 \mathrm{mN} / \mathrm{m}$ which was close to the surface tension of water.

\section{Shape and surface morphology by SEM and TEM}

The SEM pictures revealed RIF- and IH2-loaded NPs were in spherical shape and relatively monodispersed and there were no major cracks, holes or any defects seen on the surface of the formulated particles (Figures 5 and $\underline{\mathrm{S} 6}$ ). They also showed clearly that size of the particles was comparable to the value obtained from particle size analyzer. Furthermore, drug-loaded NPs were observed under TEM at high magnifications (Figure 6). The resultant shape of NPs was spherical, and a core surrounded by an outer layer 



Figure 3 Particle size distribution and zeta potential graphs of PLGA-RIF nanoparticles. Abbreviations: PLGA; poly lactic-co-glycolic acid; RIF, rifampicin.

indicated the encapsulation of drug inside the polymer matrix. The fluorescent molecule Coumarin 6 used as a model drug was encapsulated into PLGA NPs using the same procedure, and images were taken using a fluorescence microscope (Olympus). The images confirmed the spherical shape of the particles and encapsulation of the fluorescent molecule in NPs (Figure S7).

\section{Characterization of $\mathrm{N}^{\prime}$ - [phenylmethylidene] pyridine-4- carbohydrazide}

Purity: 99\% (RP-HPLC method) (Figures S8-S10). melting point: $200^{\circ} \mathrm{C}-202^{\circ} \mathrm{C}$. IR $\left(\mathrm{V}_{\max }, \mathrm{cm}^{-1}, \mathrm{KBr}\right): 3,441(-\mathrm{NH})$, 3,028 (-CH), 1,681 (amide $\mathrm{CO}$ ), 1,558 (imine $\mathrm{CN}$ ). ${ }^{1} \mathrm{H}$ NMR (400 MHz, DMSO-d6): $\delta$ ppm 7.4-7.5 (m, 2H, ArH), $7.76(\mathrm{~m}, 2 \mathrm{H}, \mathrm{ArH}), 7.83$ (d, 2H, ArH), 8.5 (s, 1H, $-\mathrm{N}=\mathrm{CH})$, 8.8 (d, 2H, ArH), 12.09 (s, 1H, -NH) (Figure 7). ${ }^{13} \mathrm{C}$ NMR (100 MHz, DMSO-d6): $\delta$ ppm 122, 127.7, 129.3, 130.8, 134.5, 140.9, 150.8, 162.09. MS: $m / z 226.09(\mathrm{MH}+)$ (Figure 8).

\section{RP-HPLC of RIF}

The chromatograms showed a sharp clear peak with a minimum tailing factor and a short runtime with RIF eluting


Figure 4 Particle size distribution and zeta potential graph of PLGA-IH2 nanoparticles.

Abbreviations: IH2, isoniazid benz-hydrazone; PLGA; poly lactic-co-glycolic acid.

at a retention time of 4.8 minutes (Figure S11). The results of statistical validation parameters such as linearity, accuracy and precision were well within their values. The peak area ratio of the drug was considered for plotting the linearity graph. The calibration curve was found to be linear in the tested concentration range of RIF. The equation of the curve was $y=30,929 x$ where " $y$ " is the peak area and " $x$ " is the

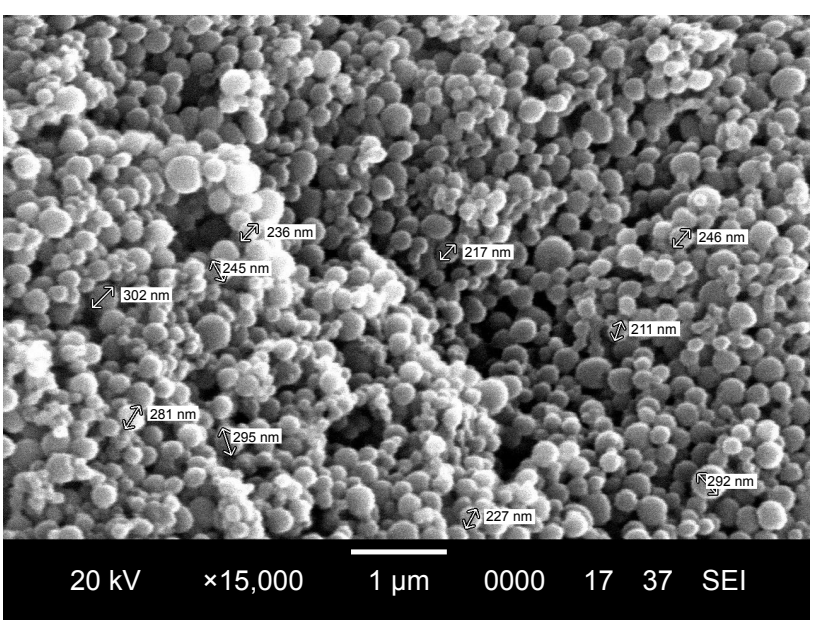

Figure 5 SEM image of IH2 encapsulated nanoparticles.

Abbreviations: $\mathrm{IH} 2$, isoniazid benz-hydrazone; SEM, scanning electron microscopy. 

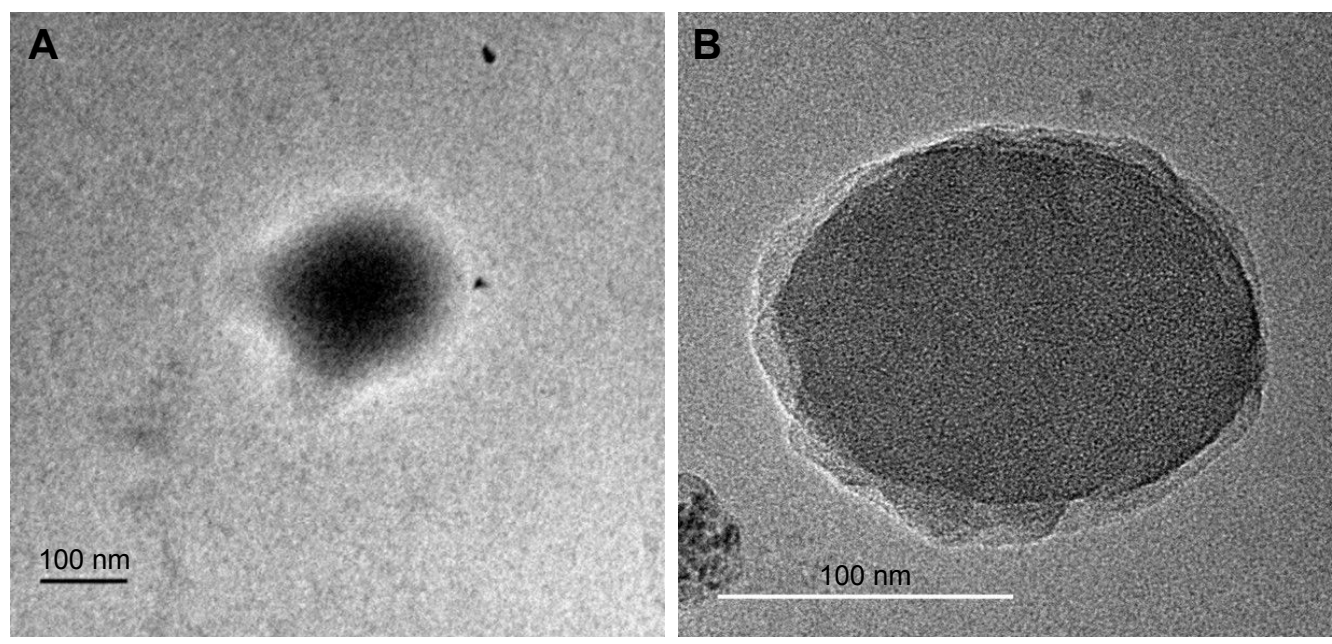

Figure 6 TEM images of (A) RIF nanoparticle and (B) IH2 nanoparticle.

Abbreviations: $\mathrm{IH} 2$, isoniazid benz-hydrazone; RIF, rifampicin; TEM, transmission electron microscopy.

concentration of drug with an $R^{2}$ value 0.9992 (Figure $\mathrm{S} 12$ ). This indicates that the developed method was significantly linear and the $\%$ relative standard deviation (RSD) of the peak area and the retention time of the drug samples were within $1 \%$ which indicated the system suitability. The $\%$ RSD values being less than $1 \%$ and retention time change at less than $0.5 \%$ indicated that the method developed is reproducible with a high precision. The LOD of RIF detectable was found to be $60 \mathrm{ng} / \mathrm{mL}$ using this method (Figure S13).

$$
\begin{aligned}
\mathrm{LOD}= & 0.06 \mu \mathrm{g} / \mathrm{mL}[3 \times(\mathrm{STDDEV} \text { of lowest } \\
& \text { concentration } / \text { Slope of calibration line })] \\
\mathrm{LOQ}= & 0.22 \mu \mathrm{g} / \mathrm{mL}[10 \times(\mathrm{STDDEV} \text { of lowest } \\
& \text { concentration } / \text { Slope of calibration line })]
\end{aligned}
$$

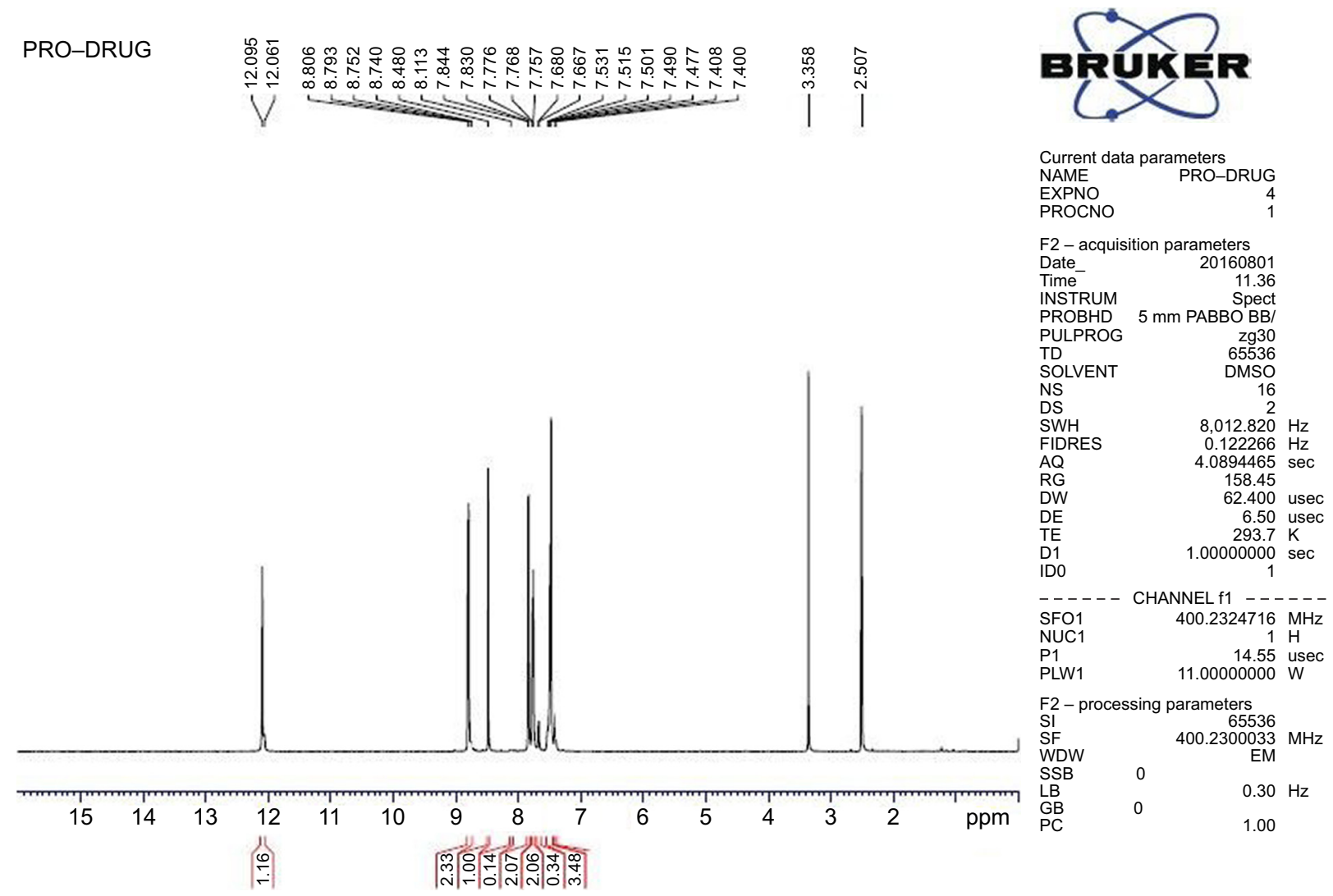

Figure $7 \mathrm{IH} 2$ characterization by 'H NMR.

Abbreviations: $\mathrm{IH} 2$, isoniazid benz-hydrazone; NMR, nuclear magnetic resonance. 


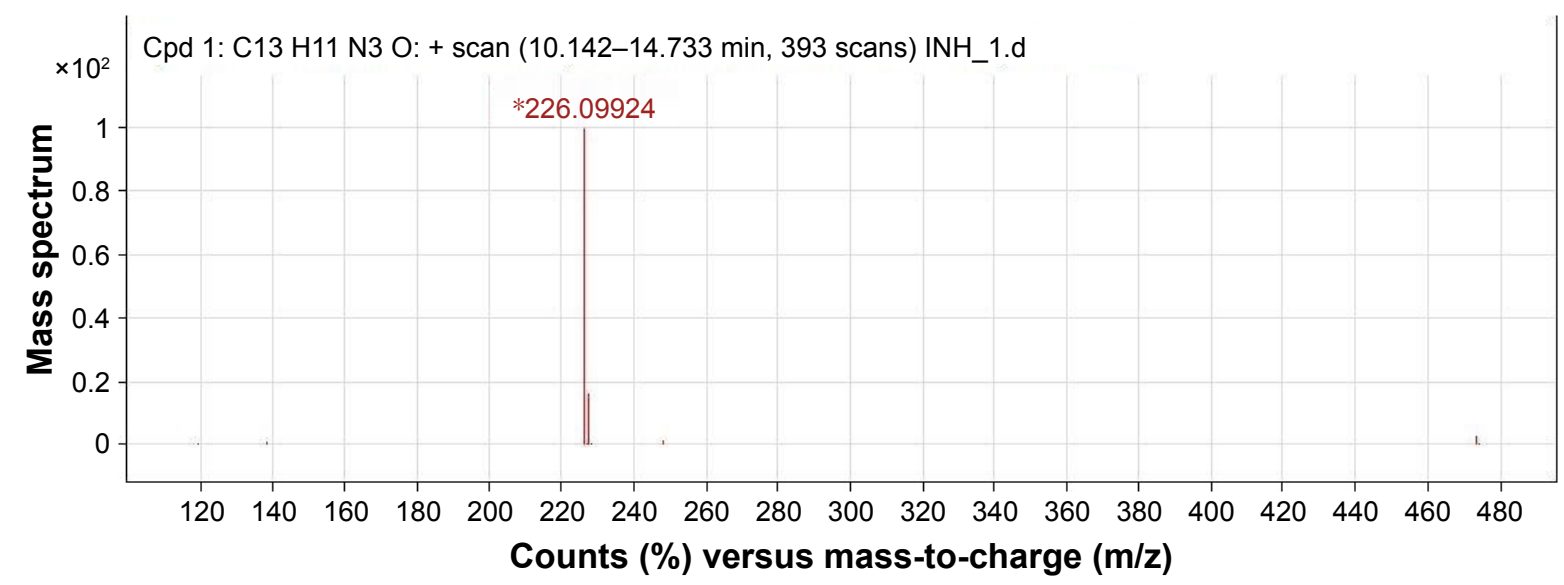

Figure 8 Mass spectrum showing molecular ion peak of $\mathrm{IH} 2$.

Note: $*=(\mathrm{M}+\mathrm{H})+$.

Abbreviations: Cpd, compound; $\mathrm{IH} 2$, isoniazid benz-hydrazone; $\mathrm{INH}$, isoniazid.

\section{$\mathrm{INH}$ and $\mathrm{IH} 2$}

In the simultaneous estimation of INH and IH2, both the components were eluted separately with different retention times. The highly hydrophilic drug INH eluted at a retention time of 4.9 minutes, and the modified hydrophobic IH2 eluted at 17.43 minutes. Both the drugs showed a clear sharp peak in HPLC chromatogram (Figure S14).

\section{Drug encapsulation in NPs}

The drug encapsulation was measured as the amount of drug in micrograms per milligram of NPs. The amount of drug encapsulated in optimized PLGA-RIF NPs was $42 \pm 7 \mu \mathrm{g} / \mathrm{mg}$ and PLGA-Coumarin NPs was $7 \pm 4 \mu \mathrm{g} / \mathrm{mg}$, and similarly, drug loading of IH2 was found to be $178 \pm 16 \mu \mathrm{g} / \mathrm{mg}$. A greater encapsulation, that is, 15 -fold more drug loading, was achieved compared to PLGA-INH NPs.

\section{Drug stability}

The stability profiles at different $\mathrm{pHs}$ were plotted as drug concentration versus time as shown in Figure 9A-C. The results indicated that stability of RIF decreased with time in all tested $\mathrm{pH}$ solutions. At $\mathrm{pH} 2.5$, RIF degraded very quickly.
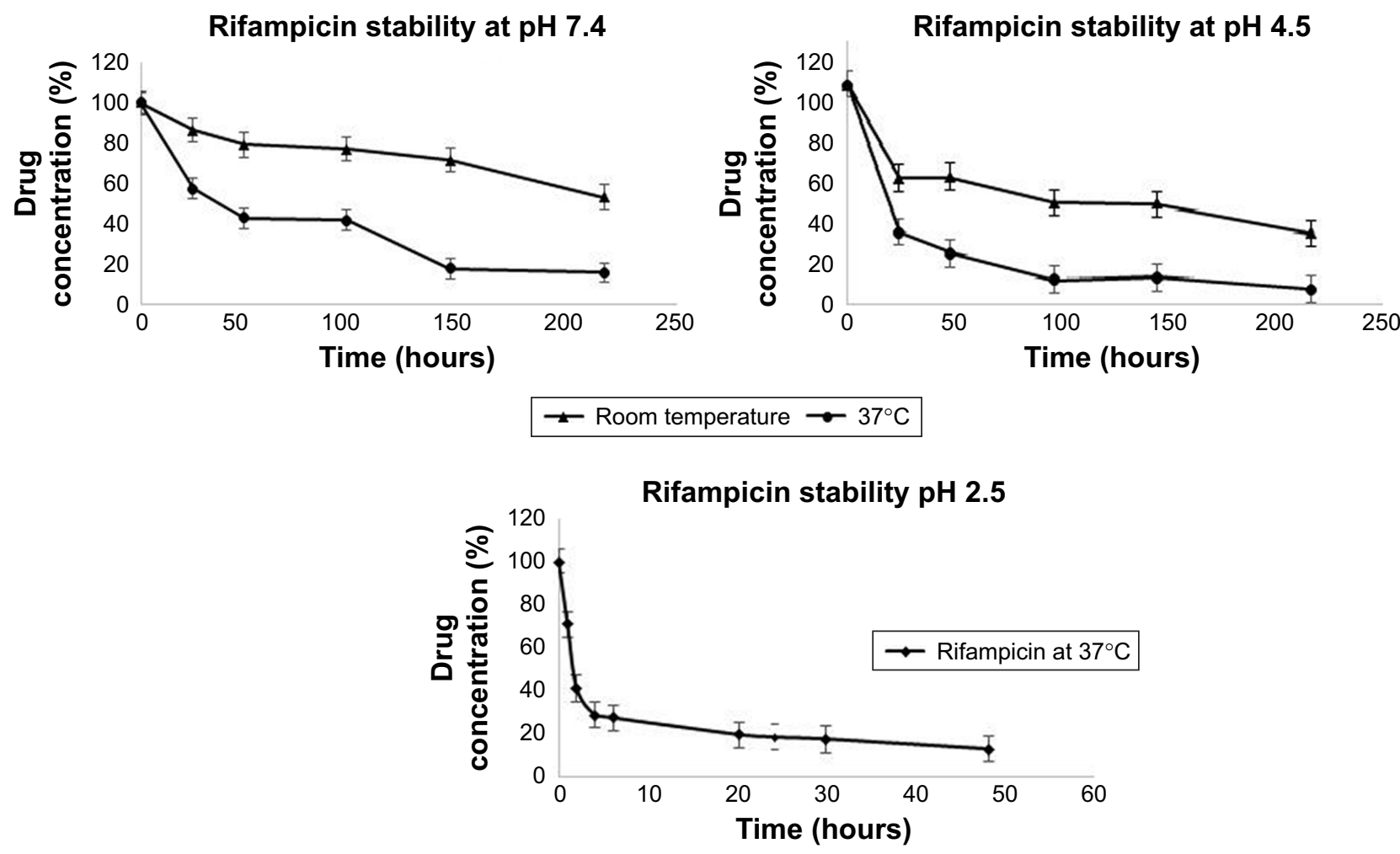

Figure 9 Rifampicin drug stability at different $\mathrm{pH}$ conditions. 


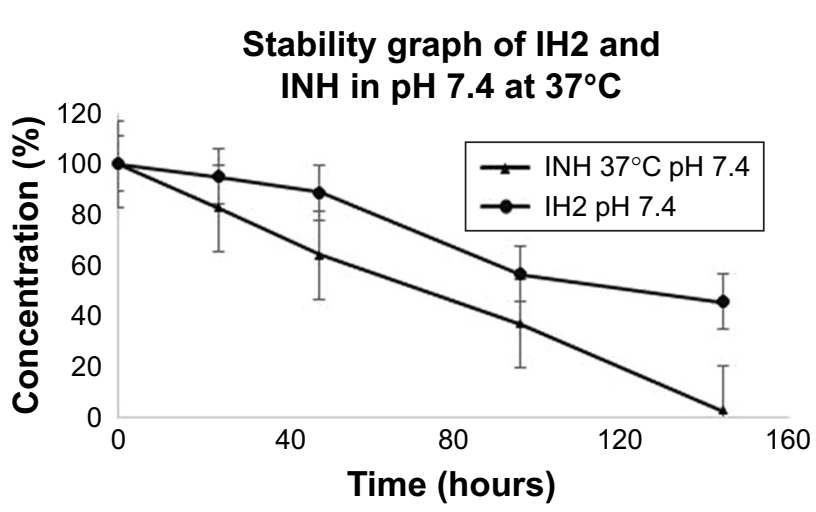

Figure $10 \mathrm{IH} 2$ and $\mathrm{INH}$ drug stability graph at $7.4 \mathrm{pH}$.

Abbreviations: $\mathrm{IH} 2$, isoniazid benz-hydrazone; $\mathrm{INH}$, isoniazid.

We observed the drastic drop in area values of initial triplicate readings of the same concentration (A1: 6975899, A2: 6219797, A3: 6023591). Almost 60\% of RIF concentration was reduced in the first 2 hours (approximately half-life of the drug in the stomach). In 24 hours, $70 \%$ of RIF degraded at $\mathrm{pH} 4.5$ and around 50\% degraded at $\mathrm{pH} 7.4$. IH2 showed good stability for up to 48 hours at both pH 4.5 (Figure S15) and 7.4, and only 5\% degradation was observed in the initial 24 hours. When stability of IH2 was compared to INH, IH2 was more stable at pH 7.4 (Figure 10).

\section{In vitro drug release}

In vitro drug release was represented by the cumulative amount of drug released in percentage with respect to time


Figure I I Drug release profile of RIF nanoparticles at different $\mathrm{pH}$ buffers. Abbreviations: PLGA, poly lactic-co-glycolic acid; RIF, rifampicin. in days or hours. The results showed an initial burst release followed by a slow and steady release of drug from the NPs. The release profile of RIF from PLGA (50:50) polymer at different $\mathrm{pH}$ conditions is shown in Figure $11 \mathrm{~A}-\mathrm{D}$, respectively.

In vitro drug release studies of $\mathrm{IH} 2$ showed similar drug release profile (Figure 12A-C).

\section{In vitro performance of PLGA-RIF and PLGA-IH2 NPs in MGITTM 960 susceptibility assay}

The Mtb culture was confirmed with acid-fast staining by the appearance of pink rod-shaped bacilli (Figure S16). Mtb was further confirmed with an MPT64 serological test. In sterility test, samples streaked on blood agar media did not show any growth for 48 hours of incubation confirming the purity of the culture (Figure S17).

The first set of experiments was carried out with $1 \mu \mathrm{g} / \mathrm{mL}$ of RIF, currently used as a standard concentration of drug, which can inhibit the growth of the H37Rv strain in a diagnostic laboratory. The control tube (H37Rv strain inoculum without any NPs) was compared with other sample tubes, that is, free form of RIF at its MIC level $(1 \mu \mathrm{g} / \mathrm{mL})$, NPs without drug and NPs encapsulated with drug equivalent to $1 \mu \mathrm{g} / \mathrm{mL}$. After incubation for $2-3$ weeks at $37^{\circ} \mathrm{C}$, control of the experiment and NPs without drug showed resistance
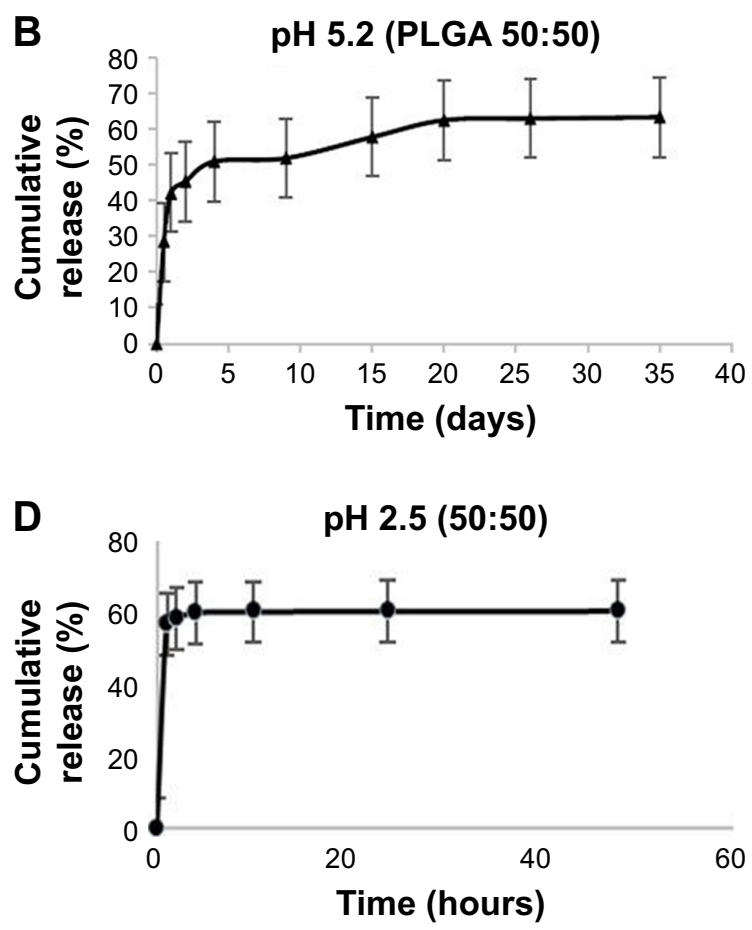

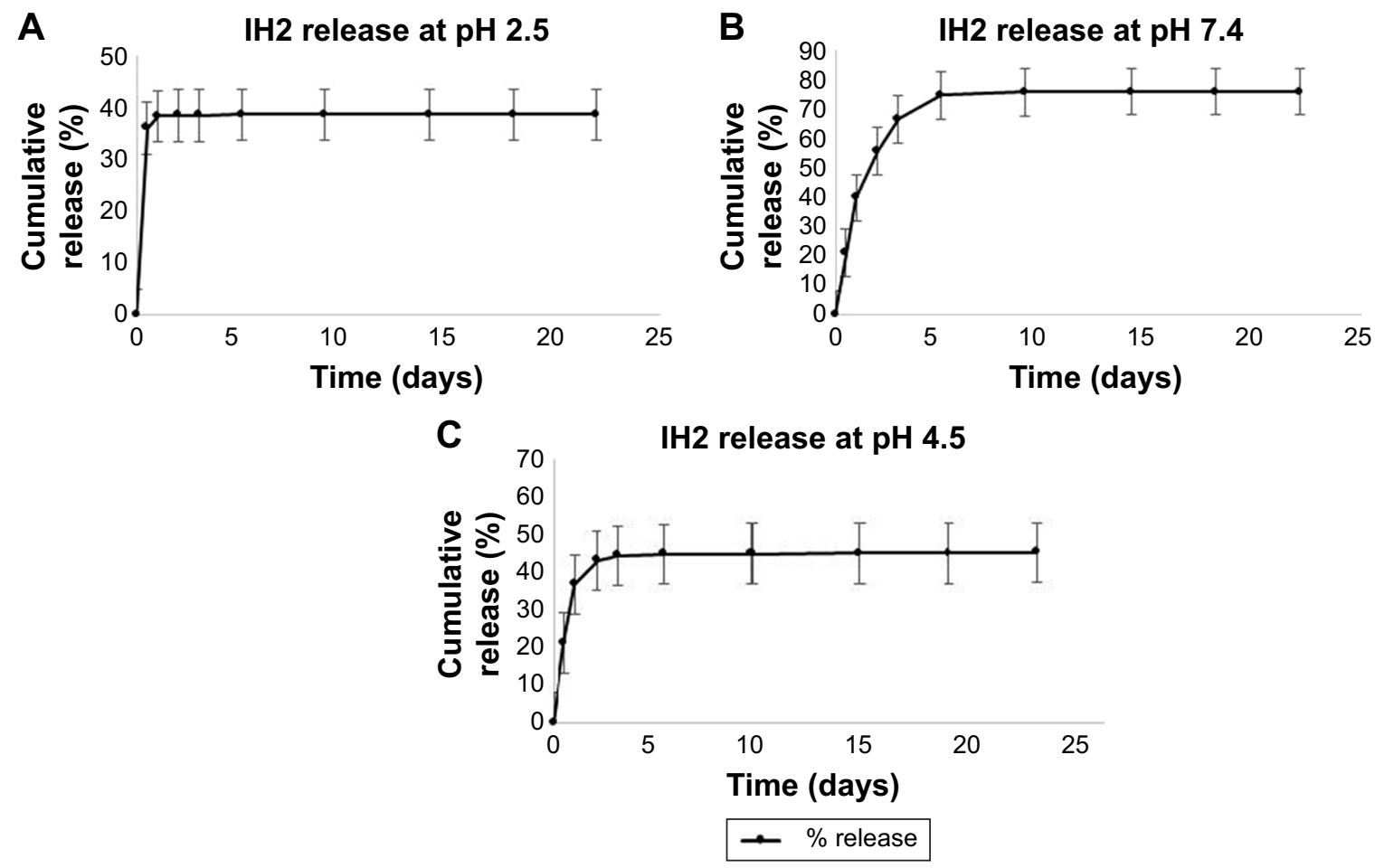

Figure 12 Drug release profile of $\mathrm{IH} 2$ nanoparticles at different $\mathrm{pH}$ buffers.

Abbreviation: $\mathrm{IH} 2$, isoniazid benz-hydrazone.

to antimicrobial susceptibility, which indicates that without any treatment and pure PLGA, NPs do not have any effect on inhibiting the growth of Mtb H37Rv strain. The RIF at its MIC level $(1 \mu \mathrm{g} / \mathrm{mL})$ was sensitive to AST which means that it inhibits the growth of H37Rv strain completely. If the concentration was reduced to less than $1 \mu \mathrm{g} / \mathrm{mL}$, RIF was resistant to AST. This indicates that pure RIF at concentration less than $1 \mu \mathrm{g} / \mathrm{mL}$ will not effectively inhibit the growth of H37Rv strain. At a reduced concentration, RIF encapsulated in NPs inhibited the growth of H37Rv strain. We obtained consistent results, and up to $0.7 \mu \mathrm{g} / \mathrm{mL}$ equivalent of RIF in NPs inhibited the growth of H37Rv strain. This proves that drugs loaded in NPs are more efficient in inhibiting the mycobacterium when compared to free drugs. Final concentration of IH2 was kept as $0.16 \mu \mathrm{g} / \mathrm{mL}$, which is the equivalent of INH MIC, that is, $0.1 \mu \mathrm{g} / \mathrm{mL}$, as part of standard procedure. The controls (without drug and NPs without drug) showed resistance to AST, while the free $\mathrm{IH} 2$ and $\mathrm{IH} 2$-encapsulated NPs inhibited the growth at MIC level, that is, $0.16 \mu \mathrm{g} / \mathrm{mL}$ (equivalent to $0.1 \mu \mathrm{g} / \mathrm{mL}$ of INH) (Table 1 ).

\section{Growth on LJ media}

After 6 weeks of incubation, control samples showed a growth of Mtb H37Rv. The tubes with appropriate treatment samples did not show any colonies. This confirmed the results obtained using MGIT $^{\mathrm{TM}}$ test.

\section{Study of cytotoxic effect of drug concentrations on A549 and RAW 264.7 cell lines}

The cytotoxic effects of drug-encapsulated NPs on A549 lung epithelial cells and RAW 264.7 macrophage cells were analyzed using MTT assays. When the cells were treated with MIC concentrations of RIF and IH 2 for 24 and 48 hours, cell viability did not change (Figure 13). These results suggest that pure drug and drug-loaded NPs have no toxic effect on

Table I Antimicrobial susceptibility test results

\begin{tabular}{lllll}
\hline $\begin{array}{l}\text { Tube } \\
\text { position }\end{array}$ & $\begin{array}{l}\text { Growth } \\
\text { unit }\end{array}$ & Status & $\begin{array}{l}\text { Drug } \\
\text { concentration }\end{array}$ & Samples \\
\hline B/MI5 & 400 & $\mathrm{C}$ & - & Growth control \\
B/MI7 & 0 & $\mathrm{~S}$ & $1.0 \mu \mathrm{g} / \mathrm{mL}$ & Free RIF \\
B/MI6 & 400 & $\mathrm{R}$ & - & NP without drug \\
B/MI8 & 0 & $\mathrm{~S}$ & $1.0 \mu \mathrm{g} / \mathrm{mL}$ & NP with RIF \\
B/M04 & 400 & $\mathrm{R}$ & $0.70 \mu \mathrm{g} / \mathrm{mL}$ & Free RIF \\
B/N09 & 0 & $\mathrm{~S}$ & $0.70 \mu \mathrm{g} / \mathrm{mL}$ & NP with RIF \\
B/JI4 & 0 & $\mathrm{~S}$ & $0.1 \mu \mathrm{gL}$ & Free INH \\
B/JI2 & 0 & $\mathrm{~S}$ & $0.16 \mu \mathrm{g} / \mathrm{mL}$ & Free IH2 \\
B/JI4 & 0 & $\mathrm{~S}$ & $0.16 \mu \mathrm{g} / \mathrm{mL}$ & NP with IH2 \\
\hline $\mathrm{Ab}$ & & $\mathrm{H}$ &
\end{tabular}

Abbreviations: C, control; $I H 2$, isoniazid benz-hydrazone; $I N H$, isoniazid; $N P$, nanoparticle; R, resistant; RIF, rifampicin; S, sensitive. 

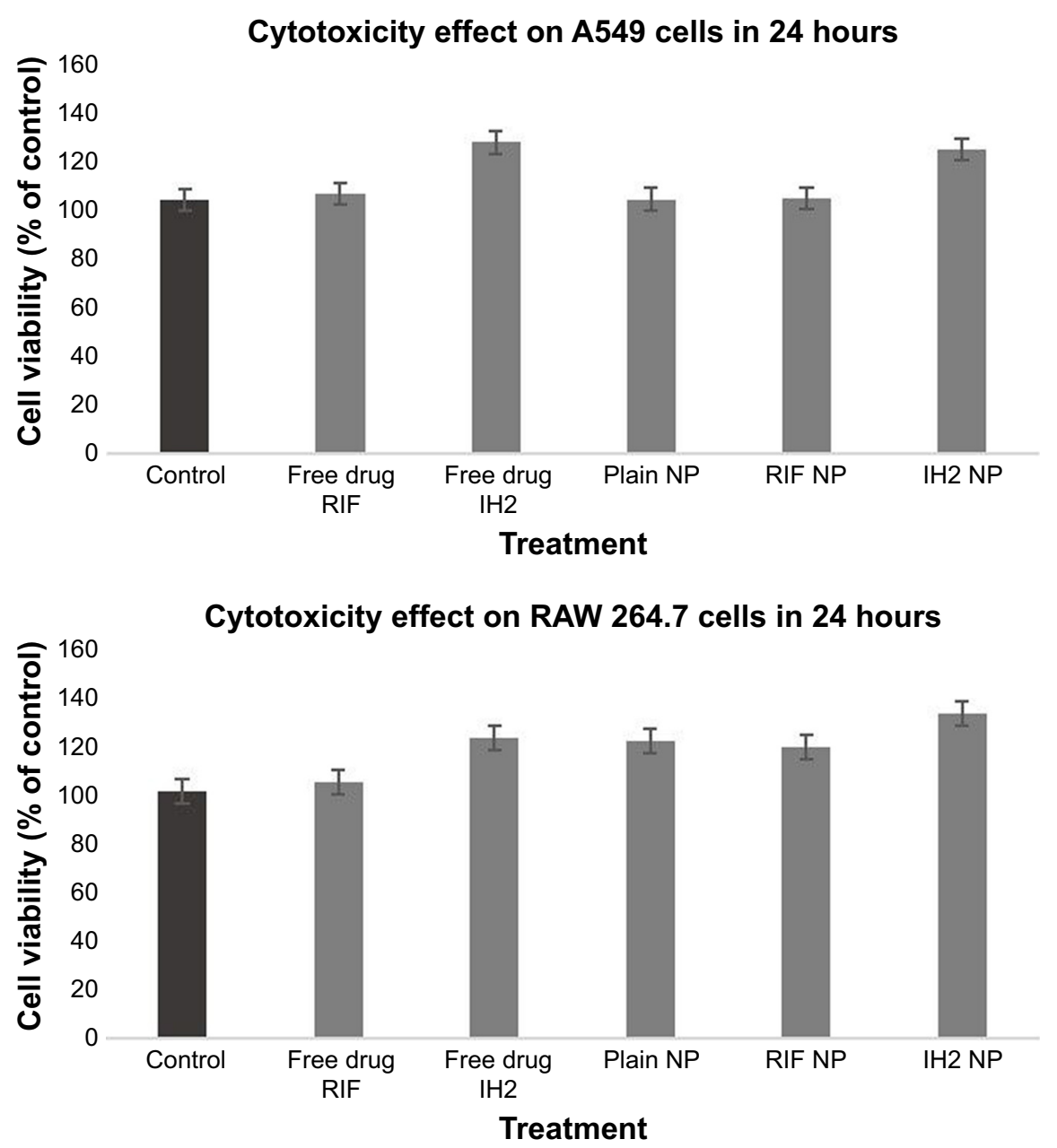

Figure 13 Cytotoxic effect on A549 cells and RAW 264.7 macrophage cells after 24 hours treatment. Abbreviations: IH2, isoniazid benz-hydrazone; NP, nanoparticle; RIF, rifampicin.

A549 lung epithelial cells and RAW 264.7 macrophage cells (48-hour results are shown in Figure S18).

\section{Discussion}

In this study, RIF- and IH2-loaded NPs and NPs without drug were formed from PLGA polymer by single-emulsion solvent evaporation method using an optimized formulation. ${ }^{34}$ PLGA 50:50 is biodegradable and hydrolytically cleavable into nontoxic oligomers or monomers of lactic acid and glycolic acid. ${ }^{40-42}$ The unique characteristics of these drugencapsulated polymeric NPs are their biocompatibility, particle size and slow and sustained release of drug. ${ }^{30}$ The surfactant used here was PVA, which helps to reduce the surface tension, thereby improving emulsion formation and drug encapsulation. ${ }^{43}$ The currently available ATDs have a half-life of certain hours which causes a major stability issue in these drugs. ${ }^{44,45}$ INH degrades and clears from the bloodstream quickly, and since INH is a highly hydrophilic drug, a very small amount of drug will penetrate through the membrane to reach the intracellular bacilli. ${ }^{46}$ This leads to an increase in the dosing frequency for patients. In contrast, the modified IH2 is hydrophobic and more stable at blood $\mathrm{pH}$. Due to hydrophobicity of IH2, more amount of drug can be encapsulated into PLGA NPs. The polymeric encapsulation provides protection from the surrounding conditions due to which the possibility of more amount of drug reaching the targeted site is enhanced. Since NPs are taken up by the cells by a different mechanism called endocytosis and able to release drug in a slow and sustained manner inside the cells, they can deliver therapeutic amount of drug to kill the intracellular bacilli. This reduces the dosing frequency of the drug and also improves the patient's compliance.

After NPs formation, removal of a major portion of PVA by washing procedure is an important step. It was done by resuspending the NPs in deionized water and centrifuging at 13,000 rpm, and the process was repeated three times to remove most of the PVA from NPs. Each time, the supernatant was collected in a separate tube, and surface tension was 
measured using a tensiometer. The surface tension of the supernatant collected from the final wash was closer to the surface tension of water - this ensured the removal of excess PVA. However, a small amount of PVA will stay associated with the NPs in spite of repeated washing due to the formation of interconnected network at the interfaces. ${ }^{47}$

The synthesized IH2 was characterized by ${ }^{1} \mathrm{H}$ NMR, ${ }^{13} \mathrm{C}$ NMR, infrared (IR) spectroscopy and MS studies. ${ }^{1} \mathrm{H}$ NMR indicated a singlet at $\delta 8.5 \mathrm{ppm}$ which was due to the $-\mathrm{N}=\mathrm{CH}-$ and a singlet at $\delta 12.09$ due to amide $-\mathrm{NH}_{2}$ indicating the formation of the product. All the other peaks were observed in expected region (Figure 7). In ${ }^{13} \mathrm{C}$ NMR, amide carbonyl was observed at $\delta 162.0 \mathrm{ppm}$ and a peak due to $-\mathrm{N}=\mathrm{CH}-$ was observed at $\delta 149.5 \mathrm{ppm}$. All the other peaks were observed in the expected region (Figure S19). The IR spectrum showed a peak at $1,681 \mathrm{~cm}^{-1}$ due to carbonyl group of amide, and a band at $1,558 \mathrm{~cm}^{-1}$ indicated the $(-\mathrm{N}=\mathrm{CH}-)$ imine bond which showed the formation of the product (Figure S20). The compound was further confirmed by mass spectral analyses, which showed $\mathrm{MH}+$ peak at $226 \mathrm{~cm}^{-1}$ (Figure 8).

The particle size analyzer is one of the most important tools to measure the distribution of NP size. The particle size distribution is effectively reported in terms of PDI. ${ }^{48}$ In DLS, if PDI is less than 0.6 , then it is considered that the particles are fairly mono-dispersed and not much aggregation of particles has taken place. In our study, PDI was less than 0.5. This indicates NPs were substantially mono-dispersed. The distribution of NPs is important because uniform distribution of particles will lead to more consistent cellular uptake, biodistribution and drug release. ${ }^{49}$ The zeta potential is measured on the basis of direction and velocity of particles under the influence of a known electric field. ${ }^{48}$ The zeta potential value characterizes the surface charge of the particles, and this in turn is used to analyze the possible extent of flocculation or de-flocculation ${ }^{50}$ and colloidal stability of the NPs. The stability of NPs is considered good if zeta potential values are $+25 \mathrm{mV}$ or $-25 \mathrm{mV} .^{51,52}$ In the present study, zeta potential of all the NPs prepared was greater than $-25 \mathrm{mV}$. This confirms that our formulations are highly stable and contain non-agglomerated NPs.

The SEM samples of NPs were coated with gold under vaccum by sputter coating before taking pictures to make them conduct and to avoid degeneration of particles due to concentrated electron beam. The SEM and TEM images clearly showed that the size and morphology of the formulated NPs were suitable for drug delivery.

The drug analysis is a very important aspect in NP drug delivery. The above-developed RP-HPLC method is simple with short retention time, indicating that large number of samples can be analyzed in a shorter time, and would be helpful in analyzing enormous number of samples (Figure S21). Significantly low LOD and LOQ values confirm the suitability of the method for analyzing lower concentration of the drug. The developed method was found to be simple, reliable, accurate and reproducible. The method was successfully applied for the quantitative analysis of drugs released from NPs and the amount of drug encapsulated in NPs.

The drug loading is an important parameter since more drug encapsulation in NPs will be beneficial for the formulation. The INH is highly water soluble, and PLGA is a hydrophobic polymer; therefore, encapsulation of INH in PLGA NPs was found to be very low. The modification of hydrophilic INH to slightly hydrophobic IH2 increased the drug loading in NPs by 15 times with the same therapeutic value as INH (Table 1). The increase in drug loading also leads to a prolonged and sustained release of therapeutic drug from the PLGA polymer and reduces dosing frequency.

The RIF, INH and IH2 were analyzed for their stability at different $\mathrm{pHs}$ using $\mathrm{pH} 7.4,4.5$ and 2.5 buffers. At $\mathrm{pH} 2.5$, RIF was found to be less stable. This result supports previous studies on RIF which showed that it degrades rapidly in an acidic condition. ${ }^{53,54}$ So, decreasing the degradation rate and increasing the bioavailability of drug is a crucial factor in the treatment of TB. The desirable characteristics of PLGA NPs offer a beneficial solution for the sustained release of drugs in the body. When we compared the results of release study and stability study, we could correlate that drug encapsulation in NPs improves the stability of the drug. Though we have shown that the release of drug at acidic $\mathrm{pH}$ is less, it is probable that some of the drugs released might have degraded and it is difficult to account for the degraded drug in HPLC. Our studies show that NPs are able to release drug in a slow and sustained manner and the drug remains considerably stable inside the NPs; therefore, NPs are suitable for delivering drugs for the treatment of TB. One of the major problems in the current treatment of TB is noncompliance to prescribed medication, mainly because treatment includes continuous and frequent multiple drug dosage. With NPs formulation, dosage frequency can be reduced. The lactide/glycolide ratio and molecular weight parameters influence drug release profile, a change that can bring about required controlled-release pattern of drug and degradation time of the PLGA. ${ }^{55,56}$ We used 50:50 PLGA with a molecular weight of 1,00,000-1,200,000 Da, and the NPs prepared using this polymer remained stable for more than 4 weeks. The stability of the pure RIF was poor in acidic $\mathrm{pH}$ (stomach condition), and RIF almost disappeared within 
48 hours and peaks were found at different retention times other than the pure drug peak in HPLC chromatogram. This shows that RP-HPLC method is very sensitive and can separate RIF from its other forms when it is degraded (Figures S22-S25, respectively). As per stability studies, more degradation of drug is the reason for less bioavailability and this in turn increases the dosage. The polymers act as a protective covering for the drugs, and NPs are taken up through cell membranes by a different mechanism called endocytosis. ${ }^{57}$ This enables NPs to reach the site of infection efficiently, and drugs are eventually released inside the cell in a slow and sustained manner over a period of time. This increases the efficacy of drug-encapsulated NPs compared to the free drug. Our result shows that drug-encapsulated NPs release drug over a period of days in acidic or neutral $\mathrm{pH}$ but the free drug degrades in hours. To the best of our knowledge, there has been no report of using drug-loaded NPs for AST using BACTEC ${ }^{\text {TM }}$ instrument. Here, the antimicrobial mixture PANTA ${ }^{\text {TM }}$ was added to reduce contamination before inoculation of the specimen for supplementing the medium. The detection of growth was based on changes in the quenching of oxygen by fluorescent dye, triggered by the oxygen consumption during the growth of mycobacteria. The culture confirmation was done with MPT64 test, with MPT64 being one of the predominant proteins found only in the culture fluid of Mtb strain. ${ }^{58}$

The results obtained from AST were further confirmed by conventional LJ re-inoculation method, and they showed that the drugs administered through NPs had a very high bioavailability compared to the free drug. The amount of drug released sustainably by NPs was good enough to show inhibition against $\mathrm{Mtb}$ in reduced concentration compared to MIC of the free drug. The adherence to treatment and the results of therapy could be made better by introducing drugencapsulated PLGA NP formulations that can release the drug in a slow and sustained manner for a considerable period.

Also, inconsistent results were seen below $0.7 \mu \mathrm{g} / \mathrm{mL}$ equivalent of RIF-encapsulated NPs. Each experiment was repeated six times, and up to $0.7 \mu \mathrm{g} / \mathrm{mL}$ equivalent of RIF-encapsulated NPs showed 100\% inhibition of growth of H37Rv strain (all six experiments resulted in no growth of H37Rv strain). Also, $0.6 \mu \mathrm{g} / \mathrm{mL}$ equivalent of RIFencapsulated NPs showed no growth in four experiments and growth of H37Rv strain in two experiments. Similarly, $0.5 \mu \mathrm{g} / \mathrm{mL}$ equivalent of RIF-encapsulated NPs showed no growth in three experiments and growth of H37Rv strain in three experiments. This might be because of small variation in the number of particles while doing serial dilution from the weighed known NPs per milliliter, which may cause small concentration variations of different samples. The possibilities of particle uptake by Mtb are unclear. This will be analyzed in further studies.

The results support that highly hydrophilic drug INH which is one of the most potent drugs against TB can be encapsulated easily inside PLGA NPs with high drug loading in an improved stable form of $\mathrm{IH} 2$ without loss of therapeutic value of the drug. The cytotoxicity results revealed that drug and drug-encapsulated NPs do not pose any toxic effect on cells. Overall, in vitro study results support drugs encapsulated in NPs are more effective than drugs in free form.

\section{Conclusion}

RIF-encapsulated PLGA NPs were synthesized and found to be substantially more effective in inhibiting the growth of H37Rv strain of Mtb compared to pure RIF. The RIF-loaded NPs inhibited the growth at a reduced concentration, that is, $0.7 \mu \mathrm{g} / \mathrm{mL}$, compared to pure RIF MIC level $(1 \mu \mathrm{g} / \mathrm{mL})$. INH was modified to IH2 to facilitate 15 times more INH equivalent encapsulation into the NPs, and when tested on H37Rv strain of Mtb, it was as effective as INH in inhibiting the growth of the strain. It was observed that ATDs are more stable when encapsulated in the NPs compared to their free form at different $\mathrm{pHs}$. The overall results support that RIF encapsulated in NPs, and INH modified to IH2 and encapsulated in PLGA NPs are more efficient in killing the H37Rv strain of Mtb.

\section{Acknowledgments}

This work was partly financed by the Vision Group of Science and Technology (VGST), State Government of Karnataka, India, under the K-FIST Level 1 awarded to Dr Bharath Raja Guru.

\section{Disclosure}

A provisional Indian patent application has been filed: Bharath Raja Guru, Santhosh L Gaonkar, Sushruta S Hakkimane and Vishnu Prasad Shenoy, inventors; Manipal Academy of Higher Education, Manipal, assignee. System and method for synthesizing isoniazid with hydrophobic moieties and encapsulating in biodegradable polymer. Indian patent 201741016102. 2018 May 7. The authors report no other conflicts of interest in this work.

\section{References}

1. Parks T, Hill AV, Chapman SJ. The perpetual challenge of infectious diseases. $N$ Engl J Med. 2012;367(1):90.

2. World Health Organisation. TB: a global emergency. Geneva: WHO; 1994. Available from: http://apps.who.int/iris/bitstream/handle/10665/58749/ WHO_TB_94.177.pdf? sequence=1\&isAllowed=y. Accessed May 1, 2014.

3. Golechha M. Can India be tuberculosis free by 2050? Lancet. 2015; 385(9965):328-329. 
4. Modlin RL, Bloom BR. TB or not TB: that is no longer the question. Sci Transl Med. 2013;5(213):213sr6.

5. World Health Organisation. Global tuberculosis report. Geneva: WHO; 2013. http://apps.who.int/iris/bitstream/handle/10665/91355/ 9789241564656_eng.pdf;jsessionid=19C24BC298D22ADE4F52713 219CACE2D?sequence=1. Accessed May 1, 2014.

6. Baxt LA, Garza-Mayers AC, Goldberg MB. Bacterial subversion of host innate immune pathways. Science. 2013;340(6133):697-701

7. Diacon AH, Patientia RF, Venter A, et al. Early bactericidal activity of high-dose rifampin in patients with pulmonary tuberculosis evidenced by positive sputum smears. Antimicrob Agents Chemother. 2007;51(8): 2994-2996.

8. Van Ingen J, Aarnoutse RE, Donald PR, etal. Why do we use $600 \mathrm{mg}$ of rifampicin in tuberculosis treatment? Clin Infect Dis. 2011;52(9):194-199.

9. Becker C, Dressman JB, Junginger HE, et al. Biowaiver monographs for immediate release solid oral dosage forms: rifampicin. J Pharm Sci. 2010;99(10):4215-4227.

10. Hartmann G, Honikel KO, Knüsel F, Nüesch J. The specific inhibition of the DNA-directed RNA synthesis by rifamycin. Biochim Biophys Acta. 1967;145(3):843-844.

11. Schwarz C, Mehnert W. Solid lipid nanoparticles (SLN) for controlled drug delivery. II. Drug incorporation and physicochemical characterization. J Microencapsul. 1999;16(2):205-213.

12. Seydel JK, Tono-oka S, Schaper KJ, Bock L, Wiencke M. Mode of action of isoniazid (INH). Short communication: isolation and identification of an analog of NAD from 3H-INH treated bacterial cells. Arzneimittelforschung. 1976;26(4):477-478.

13. Johnsson K, Schultz P. Mechanistic studies of the oxidation of isoniazid by the catalase peroxidase from Mycobacterium tuberculosis. $\mathrm{J} \mathrm{Am}$ Chem Soc. 1994;116(16):7425-7426.

14. Thiemer EK. Chemie des isoniazids [Chemistry of isoniazids]. J Ber Borstel. 1956;3:192-424. French.

15. Thiemer EK. Biochemie des isoniazids [Biochemistry of isoniazids]. $J$ Ber Borstel. 1957;4:299-509. French.

16. Seydel JK, Schaper KJ, Wempe E, Cordes HP. Mode of action and quantitative structure-activity correlation of tuberculostatic drugs of the isonicotinic acid hydrazide type. J Med Chem. 1976;19(4):483-492.

17. du Toit LC, Pillay V, Danckwerts MP. Tuberculosis chemotherapy: current drug delivery approaches. Respir Res. 2006;7(1):118.

18. Gumbo T, Louie A, Deziel MR, et al. Concentration-dependent Mycobacterium tuberculosis killing and prevention of resistance by rifampin. Antimicrob Agents Chemother. 2007;51(11):3781-3788.

19. Shishoo CJ, Shah SA, Rathod IS, Savale SS, Vora MJ. Impaired bioavailability of rifampicin in presence of isoniazid from fixed dose combination (FDC) formulation. Int J Pharm. 2001;228(1-2):53-67.

20. Singh S, Mariappan TT, Shankar R, Sarda N, Singh B. A critical review of the probable reasons for the poor/variable bioavailability of rifampicin from anti-tubercular fixed-dose combination (FDC) products and the likely solutions to the problem. Int J Pharm. 2001;228(1-2):5-17.

21. Goldman AL, Braman SS. Isoniazid: a review with emphasis on adverse effects. Chest. 1972;62(1):71-77.

22. de Faria TJ, Roman M, de Souza NM, et al. An isoniazid analogue promotes Mycobacterium tuberculosis-nanoparticle interactions and enhances bacterial killing by macrophages. Antimicrob Agents Chemother. 2012;56(5):2259-2267.

23. Monedero I, Caminero JA. MDR-/XDR-TB management: what it was current standards and what is ahead. Expert Rev Respir Med. 2009; 3(2):133-145.

24. Griffiths G, Nyström B, Sable SB, Khuller GK. Nanobead-based interventions for the treatment and prevention of tuberculosis. Nat Rev Microbiol. 2010;8(11):827-834.

25. Toti US, Guru BR, Hali M, et al. Targeted delivery of antibiotics to intracellular chlamydial infections using PLGA nanoparticles. Biomaterials. 2011;32(27):6606-6613.

26. Zhang L, Pornpattananangku D, Hu CM, Huang CM. Development of nanoparticles for antimicrobial drug delivery. Curr Med Chem. 2010; 17(6):585-594.
27. Souto EB, Muller RH. The use of SLN and NLC as topical particulate carriers for imidazole antifungal agents. Pharmazie. 2006;61(5):431-437.

28. Zhang L, Gu FX, Chan JM, Wang AZ, Langer RS, Farokhzad OC. Nanoparticles in medicine: therapeutic applications and developments. Clin Pharmacol Ther. 2008;83(5):761-769.

29. Wagner V, Dullaart A, Bock AK, Zweck A. The emerging nanomedicine landscape. Nat Biotechnol. 2006;24:1211-1217.

30. Reis CP, Neufeld RJ, Ribeiro AJ, Veiga F. Nanoencapsulation I. Methods for preparation of drug-loaded polymeric nanoparticles. Nanomedicine. 2006;2(1):8-21.

31. Chimote G, Banerjee R. Evaluation of antitubercular drug insertion into preformed dipalmitoyl-phosphatidylcholine monolayers. Colloids Surf B Biointerfaces. 2008;62(2):258-264.

32. Somoskovi A. Novel laboratory diagnostic tests for tuberculosis and their potential role in an integrated and tiered laboratory network. Tuberculosis (Edinb). 2015;95 Suppl 1:S197-S199.

33. RNTCP response to challenges of drug resistant TB in India. Revised National Tuberculosis Control Program. 2012. Available from: https:// tbcindia.gov.in/WriteReadData/1892s/8320929355Guidelines\%20 for $\% 20$ PMDT\%20in\%20India\%20-\%20May\%202012.pdf. Accessed December 1, 2016.

34. Hakkimane SS, Guru BR. Biodegradable polymer nanoparticles of anti-tubercular drug: formulation and in vitro release studies. $\mathrm{Adv} \mathrm{Sci}$ Lett. 2017;23(3):1718-1723.

35. Hakkimane SS, Guru BR. Nano formulation analysis: analytical method development of isoniazid and simultaneous estimation of anti-tubercular drugs isoniazid and rifampicin by RP-HPLC. Asian J Pharm Clin Res. 2017;10(5):330-335.

36. Patil Y, Sadhukha T, Ma L, Panyam J. Nanoparticle-mediated simultaneous and targeted delivery of paclitaxel and tariquidar overcomes tumor drug resistance. J Control Release. 2009;136(1):21-29.

37. Siddiqi SH, Rüsch-Gerdes S. MGIT ${ }^{\text {TM }}$ Procedure Manual for $B A C T E C^{\mathrm{TM}}$ MGIT $960^{\mathrm{TM}}$ TB System. Geneva: Foundation for Innovative New Diagnostics (FIND); 2006.

38. Lawson L, Emenyonu N, Abdurrahman ST, et al. Comparison of Mycobacterium tuberculosis drug susceptibility using solid and liquid culture in Nigeria. BMC Res Notes. 2013;6:215.

39. Wen ZS, Liu LJ, Qu YL, Ouyang XK, Yang LY, Xu ZR. Chitosan nanoparticles attenuate hydrogen peroxide-induced stress injury in mouse macrophage RAW264.7 cells. Mar Drugs. 2013;11(10):3582-3600.

40. Dutt M, Khuller GK. Chemotherapy of Mycobacterium tuberculosis infections in mice with a combination of isoniazid and rifampicin entrapped in poly (DL-lactide-co-glycolide) microparticles. $J$ Antimicrob Chemother. 2001;47(6):829-835.

41. Makino K, Nakajima T, Shikamura M, et al. Efficient intracellular delivery of rifampicin to alveolar macrophages using rifampicin-loaded PLGA microspheres: effects of molecular weight and composition of PLGA on release of rifampicin. Colloids Surf B Biointerfaces. 2004; 36(1):35-42.

42. Ito F, Makino K. Preparation and properties of monodispersed rifampicin-loaded poly(lactide-co-glycolide) microspheres. Colloids Surf B Biointerfaces. 2004;39(1-2):17-21.

43. Cooper DL, Harirforoosh S. Design and optimization of PLGA-based diclofenac loaded nanoparticles. PLoS One. 2014;9(1):e87326.

44. Bhandari R, Kaur IP. Pharmacokinetics, tissue distribution and relative bioavailability of isoniazid-solid lipid nanoparticles. Int J Pharm. 2013;441(1-2):202-212.

45. Behera AK, Barik BB, Pandya S, Joshi S. Formulation and evaluation of isoniazid loaded- $\sum$-polycaprolactone nanoparticles. J Pharm Res. 2012;5(2):798-802.

46. Razak SA, Yacob SFFS, Abdullah JM, Adnan R. Isoniazid and $\beta$-cyclodextrin complexes: a stability study in aqueous solution. JChem Pharm Res. 2015;7(7):346-355.

47. Sahoo SK, Panyam J, Prabha S, Labhasetwar V. Residual polyvinyl alcohol associated with poly (D L-lactide-co-glycolide) nanoparticles affects their physical properties and cellular uptake. J Control Release. 2002;82(1):105-114. 
48. Pignatello R, Ricupero N, Bucolo C, Maugeri F, Maltese A, Puglisi G. Preparation and characterization of Eudragit Retard nanosuspensions for the ocular delivery of cloricromene. AAPS PharmSciTech. 2006; 7(1):1-7.

49. Gu F, Zhang L, Teply BA, et al. Precise engineering of targeted nanoparticles by using self-assembled biointegrated block copolymers. Proc Natl Acad Sci U S A. 2008;105(7):2586-2591.

50. Zetasizer PCS. Theory manual MAN 0152. Issue 1.1: 1.1-2.6. Malvern: Malvern Instruments Ltd; 1996.

51. Wu L, Zhang J, Watanabe W. Physical and chemical stability of drug nanoparticles. Adv Drug Deliv Rev. 2011;63(6):456-469.

52. Clogston JD, Patri AK. Zeta potential measurement. Methods Mol Biol. 2011;697:63-70.

53. Shishoo CJ, Shah SA, Rathod IS, Savale SS, Kotecha JS, Shah PB. Stability of rifampicin in dissolution medium in presence of isoniazid. Int J Pharm. 1999;190(1):109-123.
54. Singh S, Mariappan TT, Sharda N, Singh B. Degradation of rifampicin isoniazid and pyrazinamide from prepared mixtures and marketed single and combination products under acid conditions. Pharm Pharmacol Commun. 2000;6:491-494.

55. Lewis DH. Controlled release of bioactive agents from lactide/glycolide polymers. In: Chasin M, Langer R, editors. Biodegradable Polymers as Drug Delivery Systems. New York: Marcel Dekker Inc; 1990:1-43.

56. Mittal G, Sahana DK, Bhardwaj V, Ravi Kumar MN. Estradiol loaded PLGA nanoparticles for oral administration: effect of polymer molecular weight and copolymer composition on release behaviour in vitro and in vivo. J Control Release. 2007;119(1):77-85.

57. Ahmad Z, Maqbool M, Raja AF. Nanomedicine for tuberculosis: insights from animal models. Int J Nano Dim. 2011;2(1):67-84.

58. Nagai S, Wiker HG, Harboe M, Kinomoto M. Isolation and partial characterization of major protein antigens in the culture fluid of $\mathrm{Myco}$ bacterium tuberculosis. Infect Immun. 1991;59:372-382.
International Journal of Nanomedicine

\section{Publish your work in this journal}

The International Journal of Nanomedicine is an international, peerreviewed journal focusing on the application of nanotechnology in diagnostics, therapeutics, and drug delivery systems throughout the biomedical field. This journal is indexed on PubMed Central, MedLine, CAS, SciSearch ${ }^{\circledR}$, Current Contents ${ }^{\circledR} /$ Clinical Medicine,

\section{Dovepress}

Journal Citation Reports/Science Edition, EMBase, Scopus and the Elsevier Bibliographic databases. The manuscript management system is completely online and includes a very quick and fair peer-review system, which is all easy to use. Visit http://www.dovepress.com/ testimonials.php to read real quotes from published authors. 